\title{
ETHzürich
}

ETH Library

\section{Effect of periodic boundary conditions on granular motion in horizontal rotating cylinders modelled using the DEM}

Journal Article

Author(s):

Third, J.R.; Scott, D.M.; Scott, S.A.; Mueller, C.R.

Publication date:

2011-02

Permanent link:

https://doi.org/10.3929/ethz-b-000030376

Rights / license:

In Copyright - Non-Commercial Use Permitted

Originally published in:

Granular Matter 13(1), https://doi.org/10.1007/s10035-010-0224-5 


\title{
Effect of periodic boundary conditions on granular motion in horizontal rotating cylinders modelled using the DEM
}

\author{
J. R. Third · D. M. Scott · S. A. Scott • C. R. Müller
}

Received: 29 June 2010 / Published online: 16 October 2010

(C) Springer-Verlag 2010

\begin{abstract}
Applying periodic boundary conditions to DEM simulations of granular motion in horizontal, rotating cylinders can result in unexpected bulk axial flow. This axial flow is shown to contribute significantly to the mean square deviation in particle position and consequently must be considered in the analysis of processes governed by slow axial motion such as axial dispersion.
\end{abstract}

Keywords Granular materials · Rotating cylinders · Discrete element method · Periodic boundary conditions

\section{Nomenclature}

$d_{\mathrm{p}} \quad$ Nominal particle diameter, $\mathrm{m}$

$d t \quad$ Timestep, $\mathrm{s}$

$D \quad$ Diameter of cylinder, $\mathrm{m}$

$f \quad$ Cylinder fill level

$F_{n} \quad$ Normal force between colliding particles, $\mathrm{N}$

$F_{t} \quad$ Tangential force between colliding particles, $\mathrm{N}$

$g \quad$ Acceleration due to gravity, $\mathrm{m} / \mathrm{s}^{2}$

$k_{n} \quad$ Normal spring stiffness, $\mathrm{N} / \mathrm{m}$

$k_{t} \quad$ Tangential spring stiffness, $\mathrm{N} / \mathrm{m}$

$L \quad$ Length of cylinder or periodic cell, $\mathrm{m}$

\section{J. R. Third $(\varangle) \cdot$ C. R. Müller}

Institute of Energy Technology, Department of

Mechanical and Process Engineering,

ETH Zürich, Leonhardstraße 27, 8092 Zürich, Switzerland

e-mail: jthird@ethz.ch

D. M. Scott

Department of Chemical Engineering and Biotechnology, University of Cambridge, Pembroke Street, Cambridge, CB2 3RA, UK

\section{S. A. Scott}

Department of Engineering, University of Cambridge, Trumpington Street, Cambridge, CB2 1PZ, UK

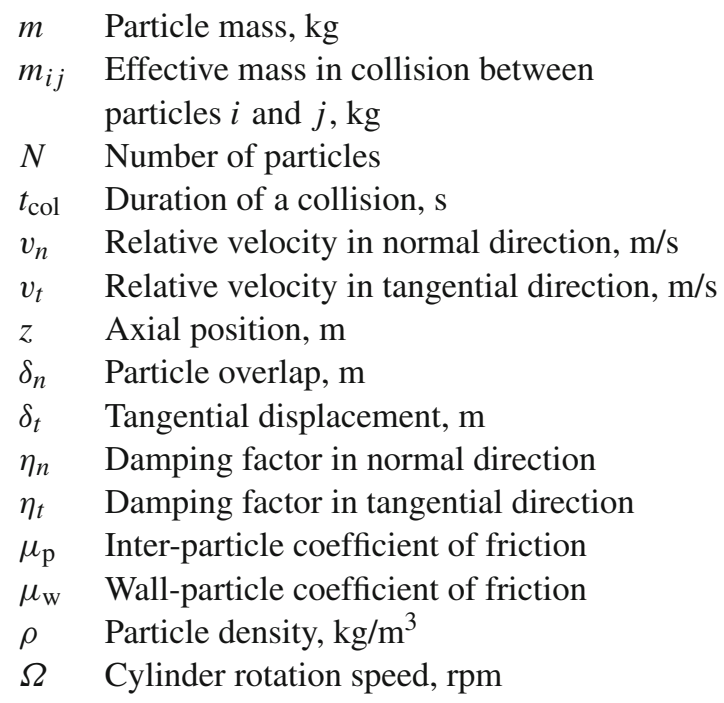

\section{Introduction}

Rotary cylinders or kilns are widely used in industry for processes such as mixing, drying, coating and performing chemical reactions. Owing to the large variety of phenomena that occur in rotating cylinders, such as radial and axial segregation or avalanching, these systems have also attracted considerable interest in the physics community. For example, Charles et al. [1] examined the effect of cylinder diameter on axial segregation patterns within rotating cylinders. Besides experimental measurements, many studies of granular motion within these cylinders have been performed using the discrete element method (DEM) [2-5]. DEM simulations of granular motion in horizontal rotating cylinders are often performed using periodic boundary conditions ( $\mathrm{PBCs}$ ) in the axial direction [2-4] since this reduces the size of the system and the computational time required. However, the use of 
PBCs in DEM simulations of rotating cylinders can result in bulk axial flow, as reported by Rapaport [2] in his study of axial segregation. Rapaport [2] showed that axial segregation can occur within periodic systems but used fixed endplates to allow a better analysis of the movement of the axial bands. This communication is to report the consequences of PBCs for the axial dispersion of approximately monosized particles within a horizontal cylinder operating within the rolling regime. Owing to the popularity of the DEM technique for modelling granular systems, we believe that these findings are of significant relevance and importance to a wider community.

\section{Simulation method}

The soft-sphere DEM is well documented in the literature [6] and will not be described here except to detail the particular force laws used in this work. In the normal direction a damped linear spring is employed and attractive forces between particles are prevented such that the force in the normal direction, $F_{n}$, for a collision between particles $i$ and $j$ is given by:

$F_{n}=\max \left(0, k_{n_{i j}} \delta_{n}-2 \eta_{n} \sqrt{\frac{m_{i j}}{k_{n}}} v_{n}\right)$

Here $\eta_{n}$ is the damping factor in the normal direction, $\delta_{n}$ is the particle overlap, $k_{n}$ is the normal stiffness, $v_{n}$ is the relative velocity in the normal direction and $m_{i j}$ is the effective mass defined as $1 / m_{i j}=1 / m_{i}+1 / m_{j}$. In the tangential direction static friction is modelled as a damped linear spring and the magnitude of the tangential force is limited by Coulomb's law such that

$F_{t}=\min \left(\mu k_{n_{i j}} \delta_{n}, k_{t_{i j}} \delta_{t}-2 \eta_{t} \sqrt{\frac{m_{i j}}{k_{t}}} v_{t}\right)$

Here $\mu$ is the coefficient of friction, $\eta_{t}$ is the damping factor in the tangential direction, $k_{t}$ is the tangential stiffness and $v_{t}$ is the relative velocity of the two surfaces in contact. The tangential displacement, $\delta_{t}$, is defined as $\int v_{t} d t$. Table 1 shows the parameter values used in this work. The fill level is defined as the fraction of the cylinder taken up by particles and voids between particles when the cylinder is rotating. The cylinder in which the particles are rotated is modelled as a smooth, but frictional, cylinder with physical properties that are identical to those of the particles. The ends of the cylinder are modelling using either PBCs or as flat, frictionless end plates. Gravity acts in a direction perpendicular to the cylinder axis for all simulations. The equations of motion are integrated using a third order Adams-Bashforth scheme with a timestep $d t$ which satisfies $d t \leq t_{\mathrm{col}} / 30$, where $t_{\mathrm{col}}$ is the duration of a collision.

The system is initialised with the particles located on a regular grid. At the start of the simulation the particles are
Table 1 Simulation parameter values

\begin{tabular}{lll}
\hline Name & Symbol & Value \\
\hline Nominal particle diameter & $d_{\mathrm{p}}$ & $3 \mathrm{~mm}$ \\
Particle size distribution & - & $\pm 5 \%$ \\
Particle density & $\rho$ & $2,500 \mathrm{~kg} / \mathrm{m}^{3}$ \\
Normal spring stiffness & $k_{n}$ & $1,000 \mathrm{~N} / \mathrm{m}$ \\
Tangential spring stiffness & $k_{t}$ & $500 \mathrm{~N} / \mathrm{m}$ \\
Normal damping factor & $\eta_{n}$ & 0.22 \\
Tangential damping factor & $\eta_{t}$ & 0.2 \\
Particle coefficient of friction & $\mu_{\mathrm{p}}$ & 0.7 \\
Acceleration due to gravity & $g$ & $9.81 \mathrm{~m} / \mathrm{s}^{2}$ \\
Cylinder diameter & $D$ & $100 \mathrm{~mm}$ \\
Length of cylinder or periodic cell & $L$ & $152 \mathrm{~mm}$ \\
Cylinder rotation speed & $\Omega$ & $10 \mathrm{rpm}$ \\
Number of particles & $N$ & 11,220 \\
Fill level & $f$ & $26 \%$ \\
Timestep for numerical scheme & $d t$ & $1 \times 10^{-5} \mathrm{~s}$ \\
\hline
\end{tabular}

given a random velocity such that the net momentum of the particles is approximately zero at $t=0$. To ensure that the system is operating in steady state, the cylinder is rotated for $20 \mathrm{~s}$ before numerical measurements are made.

\section{Results and discussion}

Within the context of rotating cylinders, axial dispersion refers to the random motion of particles along the length of the cylinder. Authors including Taberlet and Richard [5], Parker et al. [7] and Third et al. [8] have found axial dispersion within rotating cylinders to follow Fick's second law such that the mean square deviation in particle position increases linearly with time as shown by Eq. (3).

$\left\langle(z(t)-z(0))^{2}\right\rangle=2 D_{\mathrm{ax}} t$

Here $D_{\mathrm{ax}}$ is the dispersion coefficient and $z$ is the axial particle position. Except in the calculation of $D_{\mathrm{ax}}$ for systems with end caps, the mean quantities reported in this work are calculated based on all particles within the system. For systems with end caps $D_{\mathrm{ax}}$ is calculated based on the mean square deviation of a $0.1 \mathrm{~m}$ long "pulse" of particles located axially in the centre of the cylinder. Further details of this procedure are given in Third et al. [8].

Figure 1 shows the mean square deviation in particle position as a function of time for the system described in Table 1. If end caps are employed for this system then axial dispersion is found to obey Eq. (3) with $D_{\text {ax }}=1.8 \times 10^{-6} \mathrm{~m}^{2} / \mathrm{s}$. Figure 1 indicates that if $\mathrm{PBC}$ are employed the mean square deviation in particle position can be significantly larger than when end caps are applied. Furthermore, the mean square 


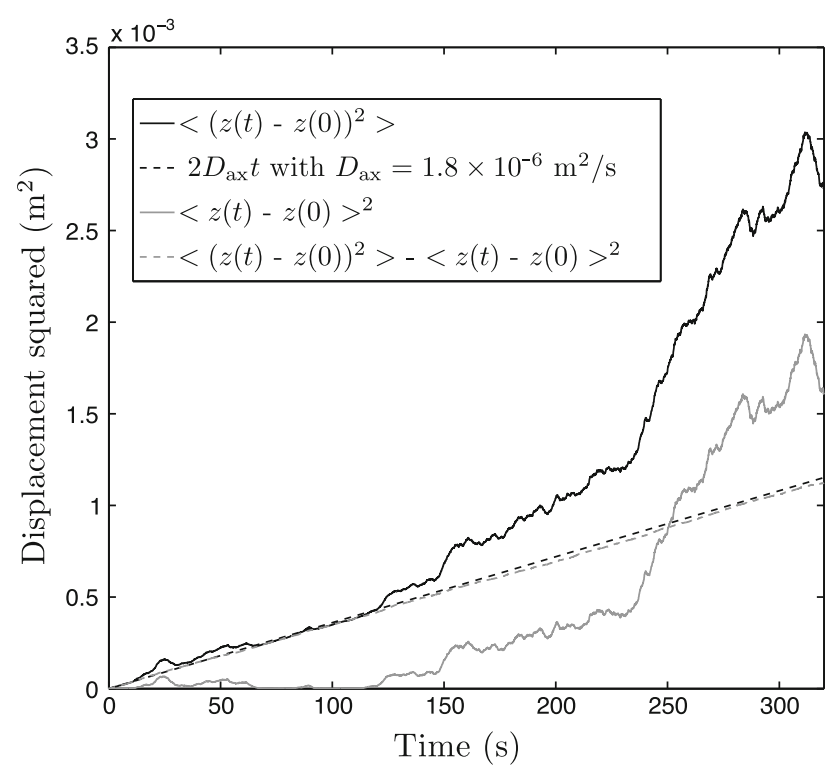

Fig. 1 Simulation results for a system with PBCs. Solid black line mean squared deviation in particle position, dashed black line Eq. (3) with $D_{\mathrm{ax}}=1.8 \times 10^{-6} \mathrm{~m}^{2} / \mathrm{s}$, solid grey line mean deviation in particle position squared, dashed grey line Eq. (4)

deviation in particle position does not increase linearly with time for the system with PBCs. This behaviour can be understood by considering the bulk axial displacement of the bed, given in Fig. 2. For the system with end caps the bulk axial displacement is approximately zero for all times, as expected. However, much larger axial displacements are observed for the system with PBCs with the result that the bulk displacement of the bed is $0.04 \mathrm{~m}$ at $t=300 \mathrm{~s}$. It should be noted that when PBCs are employed, the sustained axial velocity of the bed is small compared to the velocities of the particles in a plane perpendicular to the axis of the cylinder. For example, between $t=120$ and $t=124 \mathrm{~s}$ the bed has an average axial velocity of $0.001 \mathrm{~m} / \mathrm{s}$, whereas particles flowing down the bed surface have velocities of order $0.1 \mathrm{~m} / \mathrm{s}$.

If axial dispersion occurs independently of the bulk axial motion then the dispersive behaviour predicted by Eq. (3) can be regained by subtracting the mean displacement squared from the mean square displacement such that

$\left\langle(z(t)-z(0))^{2}\right\rangle-\langle z(t)-z(0)\rangle^{2}=2 D_{\mathrm{ax}} t$

The left-hand side of Eq. (4) is plotted as a dashed grey line on Fig. 1 and the right-hand side as a dashed black line. When the bulk motion of the bed is taken into account there is excellent agreement between the simulation performed using PBCs and the predicted behaviour based on the value of $D_{\mathrm{ax}}=1.8 \times$ $10^{-6} \mathrm{~m}^{2} / \mathrm{s}$ that was obtained from simulations using end caps. This suggests that, for this system, axial dispersion occurs independently of the bulk motion of the bed.

The data presented in Fig. 2 show net axial movement in the direction of decreasing $z$. However, the net axial dis-

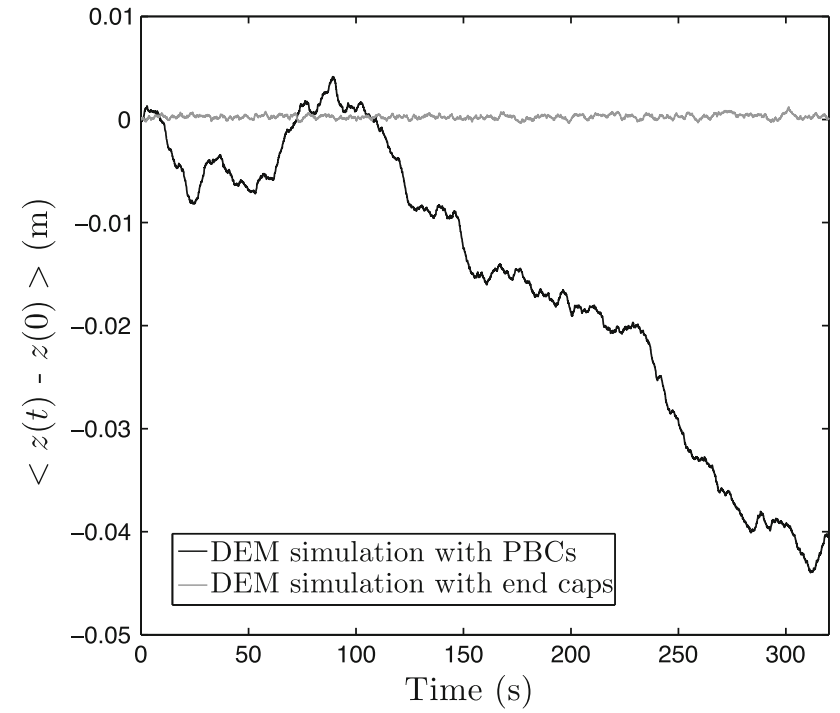

Fig. 2 Mean deviation in particle position as function of time for DEM simulations performed with end caps and $\mathrm{PBCs}$

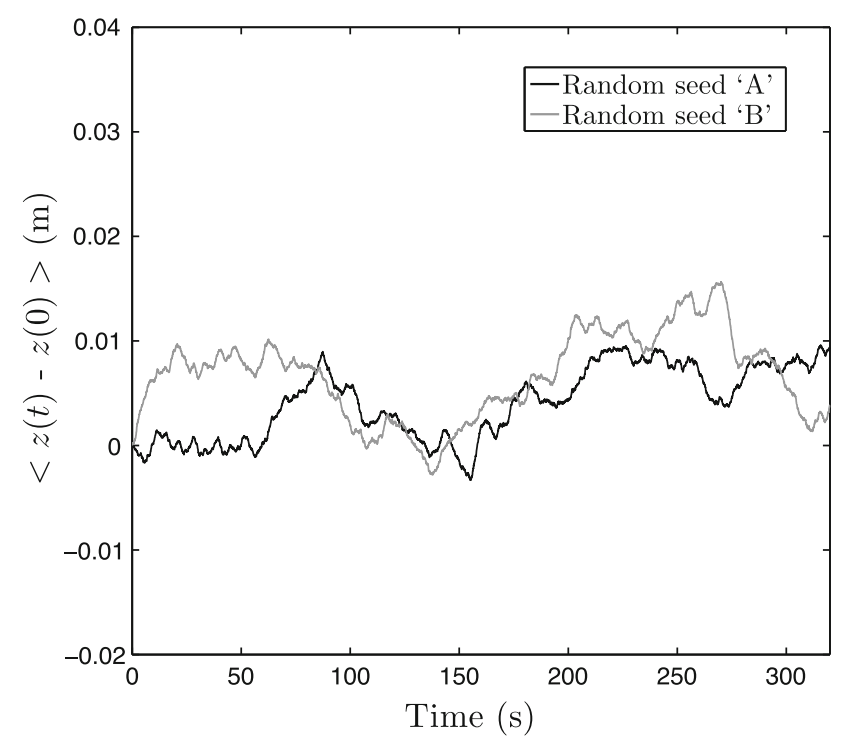

Fig. 3 Mean deviation in particle position as function of time for DEM simulations performed with PBCs for two alternative random seeds $A$ and $B$ which are different from the seed used for the calculations shown in Fig. 2

placement of this system is not a monotonic function of time, indicating that the sense of the bulk axial motion changes several times during the simulation. This suggests that this axial motion is a random process rather than the result of a continuous driving force or coding error. Further evidence of the random nature of the bulk axial motion of the bed may be obtained by repeating the simulation using different initial conditions, for example by using a different random seed. Figure 3 show the effect of changing the random seed on the mean deviation in particle position for systems with PBCs. For these data both the sense and magnitude of the net axial 
displacement are significantly different from the data shown in Fig. 2.

\section{Conclusions}

As reported by Rapaport [2], DEM simulations of rotating cylinders that employ PBCs can suffer from axial instability, leading to a bulk displacement of the bed in the axial direction. The consequences of this axial drift for axial dispersion within rotating cylinders have been highlighted here. For the system studied here, axial dispersion has been found to occur independently of the bulk motion in that the mean squared displacement is the sum of the squared mean displacement, which arises from the bulk motion, and a Fickian contribution. The results presented here demonstrate that care is required when PBCs are applied to investigate phenomena that depend on slow axial motion such as axial dispersion.

\section{References}

1. Charles, C.R.J., Khan, Z.S., Morris, S.W.: Pattern scaling in axial segregation. Gran. Mat. 8, 1-3 (2006)

2. Rapaport, D.C.: Simulation studies of axial segregation in a rotating cylinder. Phys. Rev. E 65, 061306 (2002)

3. Yang, R.Y., Zou, R.P., Yu, A.B.: Microdynamic analysis of particle flow in a horizontal rotating drum. Powder Technol. 130, 138 $146(2003)$

4. Freireich, B., Litster, J., Wassgren, C.: Using the discrete element method to predict collision-scale behavior: A sensitivity analysis. Chem. Eng. Sci. 64, 3407-3416 (2009)

5. Taberlet, N., Richard, P.: Diffusion of a granular pulse in a rotating drum. Phys. Rev. E 73, 041301 (2006)

6. Cundall, P.A., Strack, O.D.L.: A discrete numerical model for granular assemblies. Geotechnique 29, 47-65 (1978)

7. Parker, D.J., Djkstra, A.E., Martin, T.W., Seville, J.P.K.: Positron emission particle tracking studies of spherical particle motion in rotating drums. Chem. Eng. Sci. 52, 2011-2022 (1997)

8. Third, J.R., Scott, D.M., Scott, S.A.: Axial dispersion of granular material in horizontal rotating cylinders. Powder Technol. 203, 510-517 (2010)

Acknowledgments The authors would like to thank Peter Benie his advice and assistance. J.R. Third would like to thank the Engineering and Physical Sciences Research Council (EPSRC) for financial support. 